\title{
Fluid substitution in shaley sediment using effective porosity
}

\author{
Jack Dvorkin ${ }^{1}$, Gary Mavko ${ }^{1}$, and Boris Gurevich ${ }^{2}$
}

\begin{abstract}
The traditional method of fluid substitution in porous rock requires the total porosity and the elastic modulus of the mineral phase as input and assumes that the fluid reaches instantaneous hydraulic equilibrium throughout the pore space. This assumption may not be appropriate for shaley sediment because of the low permeability of shale and the resulting immobility of the water in it. To address this problem, we propose an alternative method that uses effective porosity instead of total porosity. Effective porosity is lower than total porosity if porous shale is present in the system. A new, composite mineral phase is introduced, which includes the porous water-saturated shale together with the nonporous minerals and whose elastic modulus is an average of those of its components, including the porous shale. This alternative method increases the sensitivity of the elastic properties of sedimentto-pore-fluid changes and therefore may be used as a physicsbased theoretical tool to better explain and interpret seismic data during exploration as well as variations in seismic response as hydrocarbon production progresses.
\end{abstract}

\section{INTRODUCTION}

Fluid substitution predicts the elastic properties of sediment saturated with one fluid from the properties measured when it is saturated with another fluid. It is one of the most robust tools of rock physics and is widely used in quantitative interpretation of well and seismic data - for example, for correcting sonic data for mud filtrate invasion and for computing synthetic seismograms in time-lapse seismic studies.

Typically, Gassmann's equation is used (Gassmann, 1951; Smith et al., 2003), where the bulk modulus $K_{\text {sat }}$ of the fluid saturated isotropic rock with total porosity $\phi_{t}$ is related to the moduli of the dry frame $K_{\text {dry }}$, solid phase $K_{S}$, and pore fluid $K_{F}$ as follows:

$$
K_{\mathrm{sat}}=K_{S} \frac{\phi_{t} K_{\mathrm{dry}}-\left(1-\phi_{t}\right) K_{F} K_{\mathrm{dry}} / K_{S}+K_{F}}{\left(1-\phi_{t}\right) K_{F}+\phi_{t} K_{S}-K_{F} K_{\mathrm{dry}} / K_{S}} .
$$

An implicit assumption in this equation is that the rock frame is composed of a single mineral with bulk modulus $K_{S}$. Strictly speaking, this means that Gassmann's equation cannot be used in multimineral rocks.

Brown and Korringa (1975) address this problem by generalizing the fluid substitution equation to account for the presence of different minerals in the rock frame. However, even in its simplest isotropic version, this equation requires several elastic parameters of the solid matrix that are difficult to estimate (Berryman and Milton, 1991).

Fortunately, numerical tests show that, for rocks whose minerals have elastic constants of the same order of magnitude (which is the case even for such different minerals as quartz and clay), the accuracy of Gassmann fluid substitution is more than adequate if the bulk modulus $K_{S}$ of the composite solid phase is computed using a mixing law, such as Hill's average (Hill, 1952) (e.g., Arns et al., 2002; Ciz et al., 2006). This stems from the fact that for many rocks, especially those with high porosity, $K_{S} \gg K_{\text {dry }}$ and $K_{S} \gg K_{F}$. As a result, $K_{S}$ may have only a minor influence on $K_{\text {sat }}$, which allows application of Gassmann's equation to real multimineral sediments such as sandstone, limestone, and dolomite.

Still, the use of this method for shale-rich rocks remains questionable because the presence of shale in the pore space may violate another key assumption of fluid substitution: The pore pressure fluctuations induced by a propagating stress wave must equilibrate instantaneously throughout the pore space. This is because shale contains bound water, which is essentially immobile and thus cannot be in hydraulic equilibrium with the rest of the pore space (Gurevich and Carcione, 2000).

To overcome this obstacle and to make Gassmann's equation applicable to shaley sediment, we propose an alternative approach wherein the porous wet shale is treated as part of the solid grain material. The porosity within this shale is excluded from the total porosity so that the porosity used in fluid substitution becomes the effec-

Manuscript received by the Editor September 6, 2006; revised manuscript received December 5, 2006; published online March 19, 2007

${ }^{1}$ Stanford University, Department of Geophysics, Stanford, California.E-mail: dvorkin@stanford.edu; mavko@ stanford.edu.

${ }^{2}$ Curtin University of Technology, Department of Exploration Geophysics, Perth, Western Australia and CSIRO Petroleum, Bentley, Western Australia. E-mail: boris.gurevich@geophy.curtin.edu.au.

(C) 2007 Society of Exploration Geophysicists. All rights reserved. 
tive porosity. We compare this new method with the traditional one and show that the former results in a greater sensitivity of the elastic properties of sediment-to-pore-fluid replacement. This result may be used to explain observed effects during reservoir production that cannot be justified otherwise.

\section{TRADITIONAL FLUID SUBSTITUTION}

\section{Gassmann's equation}

Consider sediment whose solid phase contains only two components, quartz and clay. Assume that the bulk modulus $K_{\text {wet }}$ of rock at full water saturation $\left(S_{W}=1\right)$ is known. The goal is to calculate the compressional modulus $K_{\text {sat }}$ of the same rock at partial water saturation $\left(S_{W}<1\right)$ where part of the pore space is filled with hydrocarbon.

The value $K_{\text {wet }}$ can be calculated from the measured $V_{p}, V_{s}$, and bulk density $\rho_{b}$. If $V_{s}$ is neither available nor reliable, an approximate $V_{p}$-only fluid substitution can be used (Mavko et al., 1995), which uses the compressional modulus $M=K+(4 / 3) \mu$, where $\mu$ is the shear modulus, instead of the bulk modulus $K$ (Appendix A).

Fluid substitution includes two steps. The first is to calculate the dry-frame bulk modulus:

$$
K_{\mathrm{dry}}=K_{S} \frac{1-\left(1-\phi_{t}\right) K_{\mathrm{wet}} / K_{S}-\phi_{t} K_{\mathrm{wet}} / K_{W}}{1+\phi_{t}-\phi_{t} K_{S} / K_{W}-K_{\mathrm{wet}} / K_{S}} .
$$

The second is to use equation 1 to derive $K_{\text {sat }}$, where $K_{F}$ is now the effective modulus of the mixture of water and hydrocarbon at $S_{W}<1$.

The elastic moduli $K_{F}$ and $K_{S}$ in these equations must be calculated from the moduli of the constituents of the fluid and solid phases, respectively.

\section{Effective bulk modulus of the fluid phase}

If two or more fluid phases are present in the pore space, $K_{F}$ is the isostress (or harmonic) average of the bulk moduli of the components and is calculated from Wood's equation (Wood, 1941; White, 1983):

$$
K_{F}=\left(\sum_{i=1}^{n} S_{i} K_{F i}^{-1}\right)^{-1},
$$

where $n$ is the number of fluid-phase components, $S_{i}$ is the volume fraction of the $i$ th component of the fluid phase, and $K_{F i}$ is the bulk modulus of $i$ th component. This mixing law is justified by the assumption that the pressure (stress) variations triggered in individual fluid components by a seismic wave are the same for all components. This assumption implies that the saturation pattern in the rock is uniform, i.e., the pore-fluid components coexist on such a fine scale that the pressure variations in these components have sufficient time to equilibrate during the wave period.

If the fluid mixture includes only two components, water and hydrocarbon with volume fractions $S_{w}$ and $1-S_{w}$, respectively, the effective bulk modulus of their mixture is

$$
K_{F}=\left[S_{W} K_{W}^{-1}+\left(1-S_{W}\right) K_{H}^{-1}\right]^{-1},
$$

where $K_{H}$ is the bulk modulus of the hydrocarbon.

\section{Effective bulk modulus of the solid phase}

The effective elastic moduli of a composite that includes several elastic components depend on the moduli of those components as well as their spatial arrangement. The range of possible variations of these effective elastic moduli for an isotropic composite lies between the lower and upper Hashin-Shtrikman bounds (Hashin and Shtrikman, 1963). These bounds are within a wider range, which is defined by the Reuss lower bound and the Voigt upper bound (Mavko et al., 1998).

The lower bound is the harmonic average of the elastic moduli of individual components of a composite, and the upper bound is their arithmetic average. It is common practice to estimate the effective elastic moduli of the composite $\left(M_{S}, \mu_{S}\right.$, and $\left.K_{S}\right)$ as the arithmetic average of the Reuss and Voigt bounds:

$$
\begin{aligned}
& K_{S}=\frac{1}{2}\left[\sum_{i=1}^{m} f_{S i} K_{S i}+\left(\sum_{i=1}^{m} f_{S i} K_{S i}^{-1}\right)^{-1}\right], \\
& \mu_{S}=\frac{1}{2}\left[\sum_{i=1}^{m} f_{S i} \mu_{S i}+\left(\sum_{i=1}^{m} f_{S i} \mu_{S i}^{-1}\right)^{-1}\right],
\end{aligned}
$$

and

$$
M_{S}=K_{S}+\frac{4}{3} \mu_{S}
$$

where $K_{S i}$ and $\mu_{S i}$ are the bulk and shear moduli, respectively, of the $i$ th elastic component whose volume fraction in the composite is $f_{S i}$ and where $m$ is the number of components.

Now consider sediment whose solid phase contains only two components, quartz and clay, and apply the bulk and shear moduli of quartz, 36.6 and $45 \mathrm{GPa}$, respectively, and of clay, 21 and $7 \mathrm{GPa}$, respectively (Mavko et al., 1998). The effective bulk and compressional moduli of this quartz and clay mixture, according to equations 5-7 as well as the Hashin-Shtrikman and Voigt-Reuss elastic bounds, are plotted in Figure 1.

In spite of the large elastic contrast between quartz and clay, the elastic bounds are fairly close to each other and to Hill's average. This implies that the error possibly introduced into fluid substitution results by using Hill's average may not be critical.

Still, it is important to remember that equation 7 is just one of several mixing laws that can be used in fluid substitution. In specific situations, such as where the softer component of the solid frame (usually clay) is load bearing, the use of the lower Hashin-Shtrikman bound may be more appropriate (Goldberg and Gurevich, 1998).

\section{ALTERNATIVE FLUID SUBSTITUTION FOR SHALEY SAND}

\section{Volume balance for porosity and saturation}

Consider a porous rock with clay (Figure 2). The volume fraction of the clay mineral in the whole mineral phase is $f_{\text {clay }}$. The intrinsic porosity of clay (the microporosity) is $\phi_{\text {clay }}$. In a unit volume of rock, the volume occupied by the nonclay minerals (assumed to be nonporous $)$ is $\left(1-f_{\text {clay }}\right)\left(1-\phi_{t}\right)$. The volume occupied by the clay mineral is $f_{\text {clay }}\left(1-\phi_{t}\right)$. Then the volume occupied by the porous clay is 


$$
C=\frac{f_{\text {clay }}\left(1-\phi_{t}\right)}{1-\phi_{\text {clay }}} .
$$

The total void space in the rock is the sum of the void space outside the porous clay and that within the clay. The void space outside the porous clay (in a unit volume of rock) is the effective porosity $\phi_{e}$ :

$$
\phi_{e}=\phi_{t}-\phi_{\text {clay }} C=\phi_{t}-f_{\text {clay }} \phi_{\text {clay }} \frac{1-\phi_{t}}{1-\phi_{\text {clay }}} .
$$

Let us introduce a modified solid phase that includes the nonclay minerals plus the porous clay. The volume of this modified solid phase in a unit volume of rock is $1-\phi_{e}$. The volume fraction of the porous clay in the modified solid is

$$
f_{\text {Pclay }}=\frac{f_{\text {clay }}}{\left(1-\phi_{\text {clay }}+\phi_{\text {clay }} f_{\text {clay }}\right)} .
$$

Assume next that part of the pore space in the rock is saturated with hydrocarbon whose volumetric fraction in the whole pore volume is $S_{H}=1-S_{W}$. Assume also that the intrinsic pore space of the clay is fully water saturated and all hydrocarbon is contained in the effective pore space. Then the volumetric fraction of hydrocarbon in the effective pore space is

$$
S_{H e}=\phi_{t}\left(1-S_{W}\right) / \phi_{e}
$$

and the volumetric fraction of water in the effective pore space (or effective water saturation) is

$$
S_{W e}=1-S_{H e}=1-\phi_{t}\left(1-S_{W}\right) / \phi_{e} .
$$

As expected, $S_{W e}$ becomes zero if the only water in the rock is that contained inside the clay, i.e., $S_{W}=C \phi_{\text {clay }} / \phi_{t}$.

\section{Gassmann's equation with effective porosity}

Let us now substitute fluid merely in the effective pore space (rather than in the total pore space) while treating the mixture of quartz and wet clay as a new composite solid. This can be done similarly to the traditional method described by equations 1 and 2 but replacing $\phi_{t}$ with $\phi_{e}$ and altering the bulk moduli of the solid and fluid.

In particular, the dry-frame modulus of the rock, which in this case is the modulus of the rock where the effective pore space is empty, is

$$
K_{\text {drye }}=K_{S e} \frac{1-\left(1-\phi_{e}\right) K_{\mathrm{wet}} / K_{S e}-\phi_{e} K_{\mathrm{wet}} / K_{W}}{1+\phi_{e}-\phi_{e} K_{S e} / K_{W}-K_{\mathrm{wet}} / K_{S e}},
$$

while the bulk modulus of the same rock at partial water saturation is

$$
K_{\text {sat }}=K_{S e} \frac{\phi_{e} K_{\text {drye }}-\left(1-\phi_{e}\right) K_{F e} K_{\text {drye }} / K_{S e}+K_{F e}}{\left(1-\phi_{e}\right) K_{F e}+\phi_{e} K_{S e}-K_{F e} K_{\text {drye }} / K_{S e}},
$$

where $K_{\text {drye }}, K_{S e}$, and $K_{F e}$ are the bulk moduli of the dry frame, solid, and pore fluid, respectively, to be used in this alternative method of fluid substitution. These moduli differ from the $K_{\mathrm{dry}}, K_{S}$, and $K_{F}$ parameters used in equations 1 and 2.

Specifically, water and hydrocarbon must now be mixed inside the effective pore space. The resulting bulk modulus (using the harmonic mixing law) is

$$
K_{F e}=\left[S_{W e} K_{W}^{-1}+\left(1-S_{W e}\right) K_{H}^{-1}\right]^{-1},
$$

where $S_{W e}$ is given by equation 12 .

The solid now includes (in the case of a quartz and clay sediment) quartz and porous water-filled clay. The effective bulk modulus of this composite solid can be computed as Hill's average according to equations 5-7, with $m=2, f_{S 1}=1-f_{P \text { clay }}, f_{S 2}=f_{P \text { clay }}, K_{S 1}=K_{Q}$, $K_{S 2}=K_{P \text { clay }}, \mu_{S 1}=\mu_{Q}$, and $\mu_{S 2}=\mu_{P \text { clay }}$ and with $f_{P \text { clay }}$ given by equation 10 . The subscript $Q$ refers to the constants for quartz. As yet
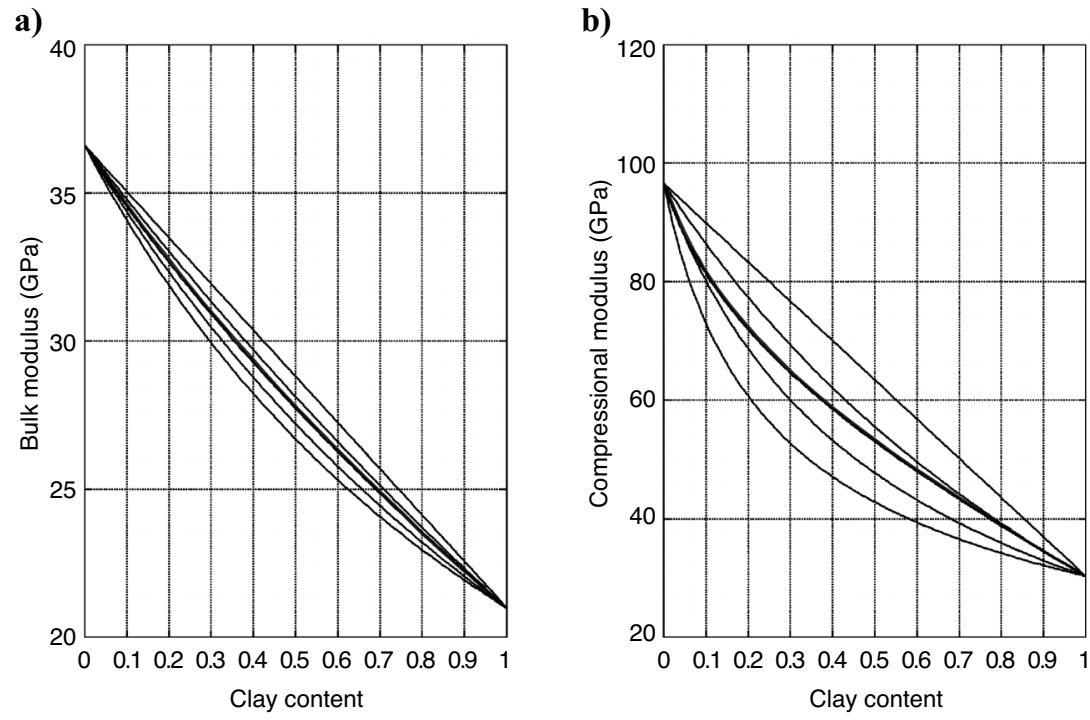

Figure 1. Relationships of (a) effective bulk modulus and (b) compressional modulus with clay content of a quartz/clay mixture. The bold curve in the middle is calculated trikman bounds (Hashin and Shtrikman, 1963). The outer two curves represent the Voigt and Reuss bounds (Mavko et al., 1998).

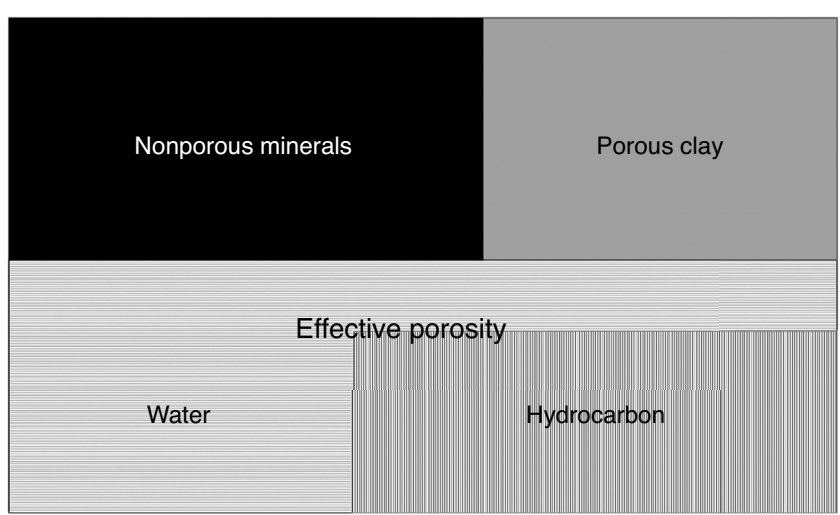

Figure 2. Schematic representation of the porosity components of a reservoir rock. The effective pore space contains water and hydrocarbon. The total pore space includes the effective pore space plus that inside the porous clay. 
unknown are $K_{P_{\text {clay }}}$ and $\mu_{P \text { clay }}$, the bulk and shear moduli of the wet porous clay.

These moduli depend on the intrinsic porosity of the clay $\left(\phi_{\text {clay }}\right)$ in the pore space as well as on the effective pressure, the texture and mineralogy of the clay, and the bulk modulus of the brine. One way of estimating these moduli is by using those of a $100 \%$ clay interval, provided that such an interval can be found in the well under examination. Another way is by using a rock physics model that links the moduli of porous clay to its intrinsic porosity and mineralogy. Several such models are available. One that is appropriate for mature consolidated sediment is described by Raymer et al. (1980). Another, relevant to soft geologically young clay, is the uncemented (soft) sand/shale model of Dvorkin and Nur (1996).

Figure 3 shows that, depending on the geological setting, the shale data may fall onto one of these model curves. The choice of the mod$\mathrm{el}$, as well as the porosity and mineralogy of the clay, is somewhat arbitrary but still must be geologically consistent and site specific. A range of these inputs can be considered to obtain a potential range of responses to fluid alteration in shaley sand. (Recently acquired laboratory data on velocity in shales and relevant models can be found in Holt and Fjaer [2003] and Holt et al. [2004].)

\section{EXAMPLE}

Consider wet rock where the elastic moduli are related to porosity and mineralogy according to the uncemented sand/shale model of Dvorkin and Nur (1996). The bulk and shear moduli of quartz used in this model are 36.6 and $45 \mathrm{GPa}$, respectively; those for the clay

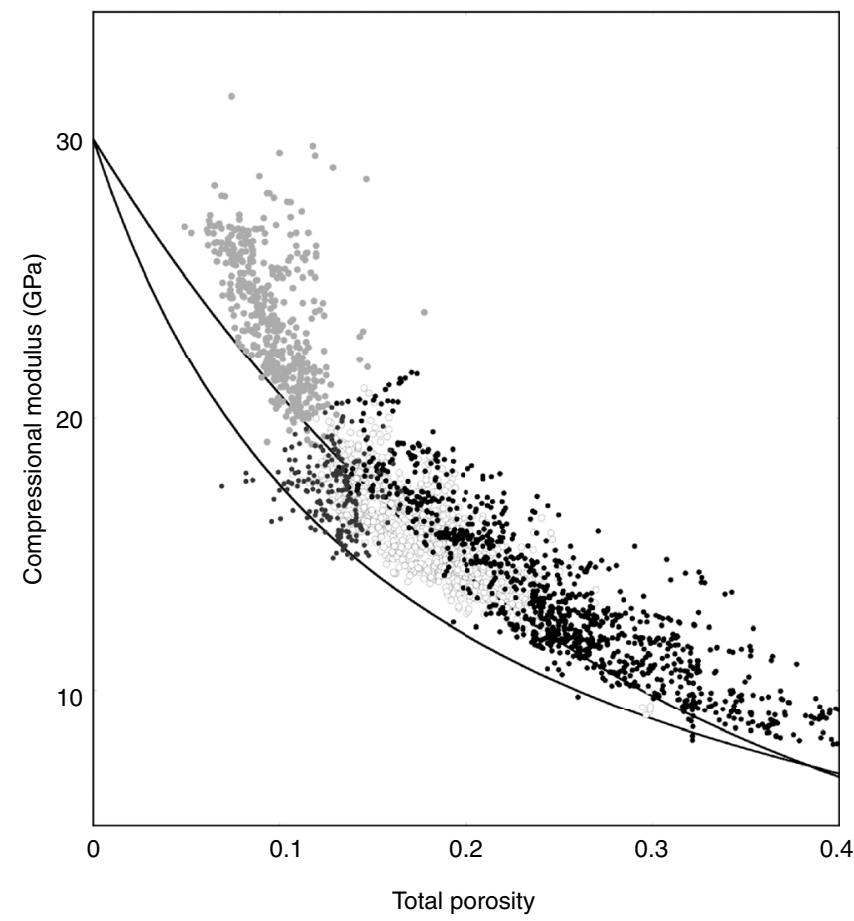

Figure 3. The relationship between compressional modulus of a water-saturated shale and total porosity. Different symbols indicate different wells. The upper curve is from the model of Raymer et al. (1980), and the lower curve is from the soft sand/shale model of Dvorkin and Nur (1996). The model curves are calculated for shale with $100 \%$ clay content. Some of the data points lie above the upper curve because their mineralogy includes quartz and feldspar. are 21 and $7 \mathrm{GPa}$, respectively. The density of quartz is $2.65 \mathrm{~g} / \mathrm{cm}^{3}$, while that of the clay mineral is $2.58 \mathrm{~g} / \mathrm{cm}^{3}$.

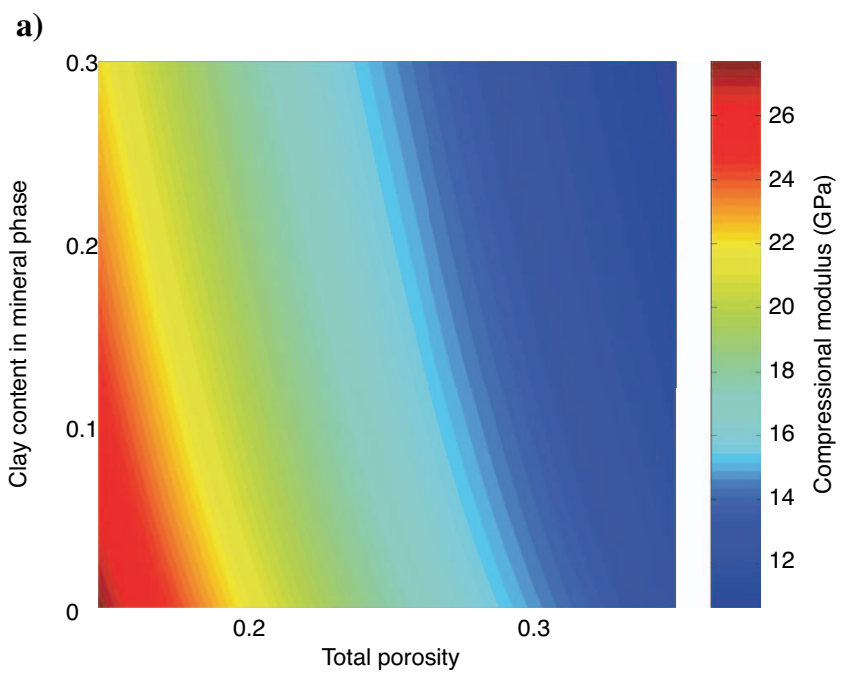

b)
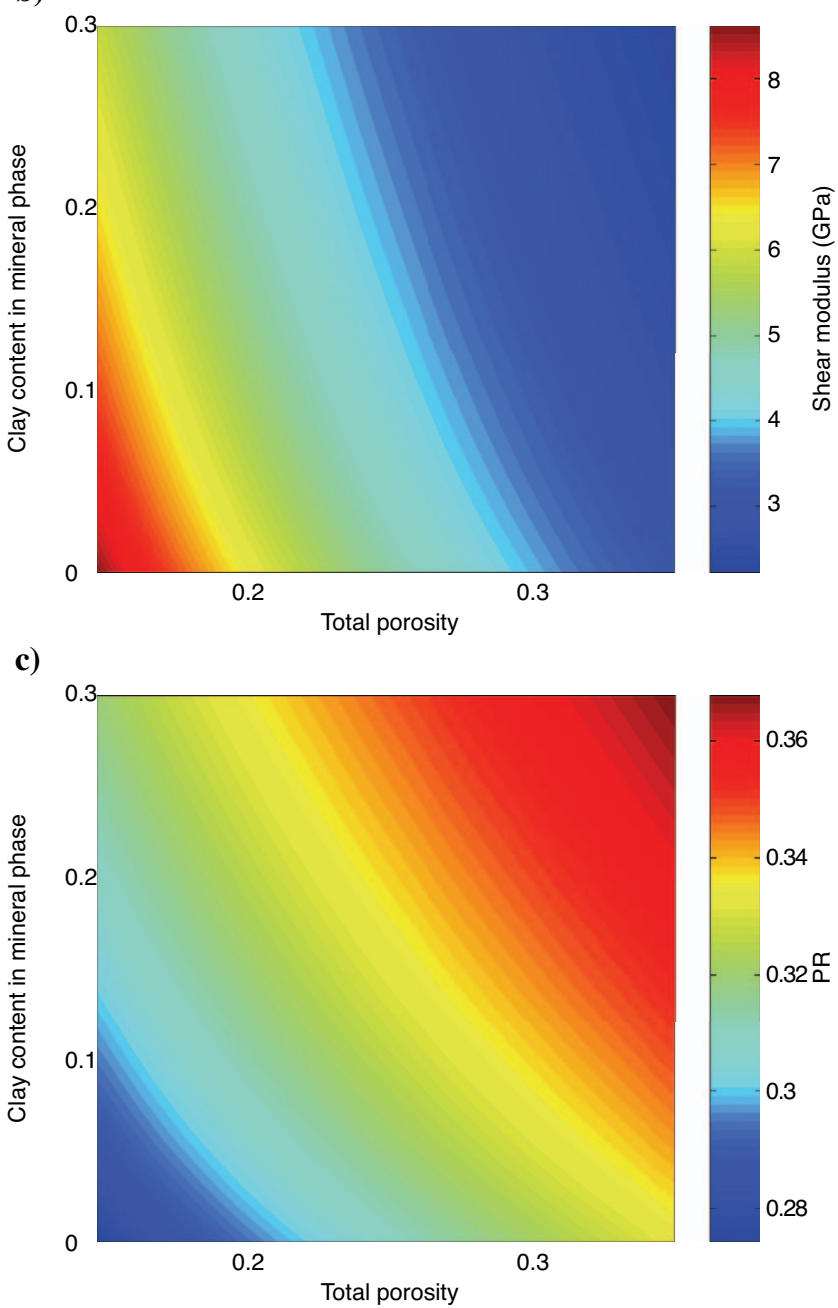

Figure 4. (a) Compressional modulus, (b) shear modulus, and (c) Poisson's ratio in a fully water-saturated sand (the soft sand/shale model; Dvorkin and Nur [1996]) for a range of porosities and clay contents for the example discussed in the text. 
a)

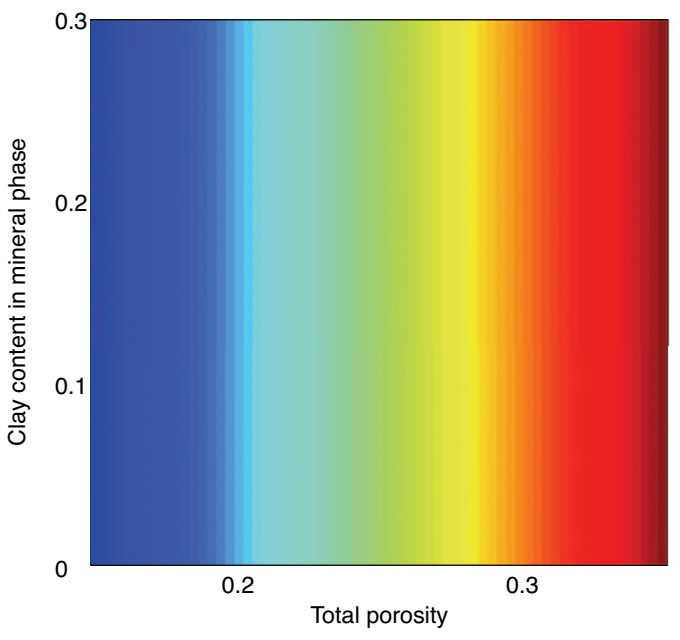

b)

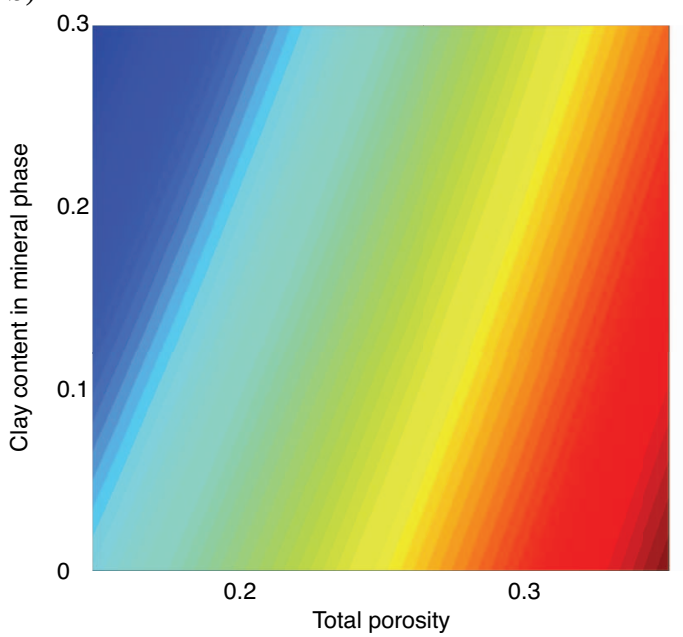

c)

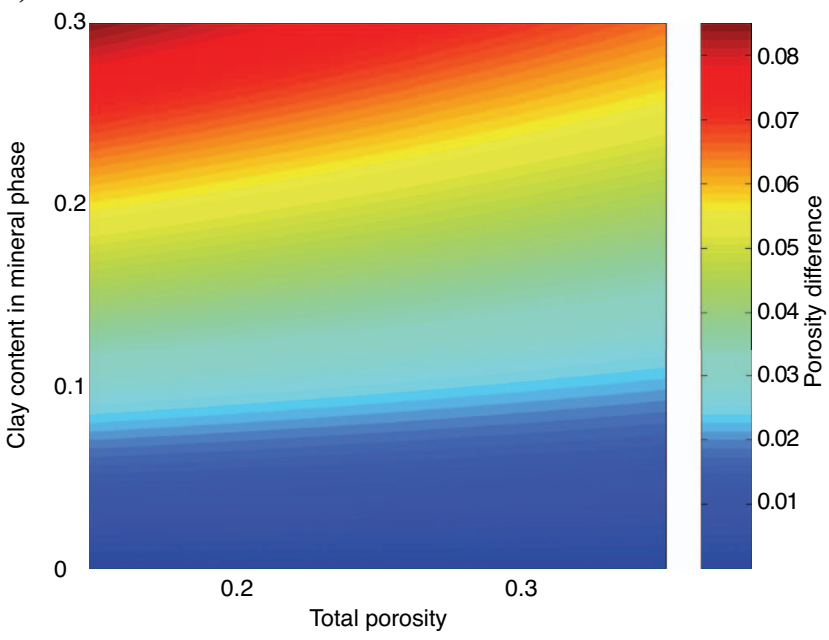

Figure 5. (a) Total porosity, (b) effective porosity, and (c) their difference for a range of clay contents for the example discussed in the text. a)

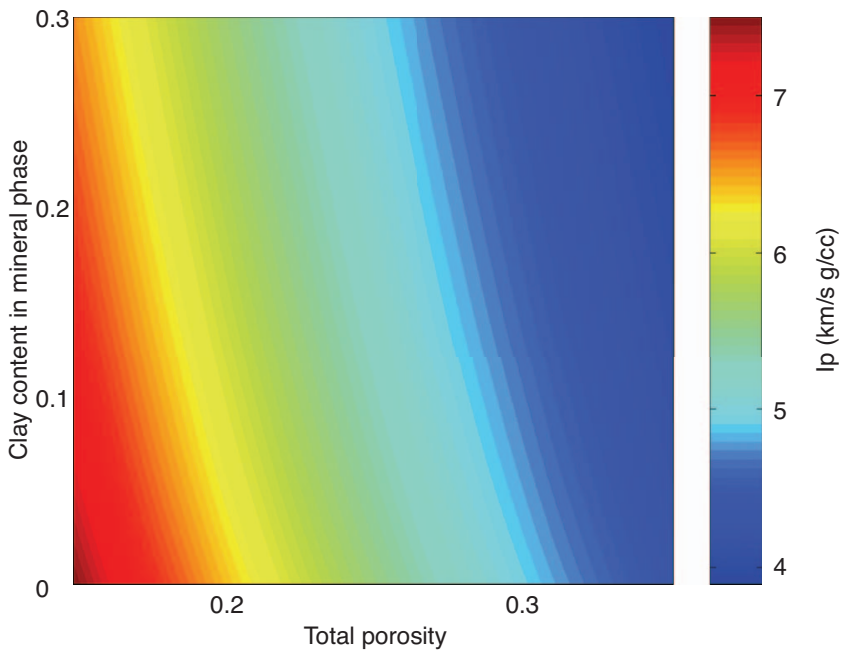

b)

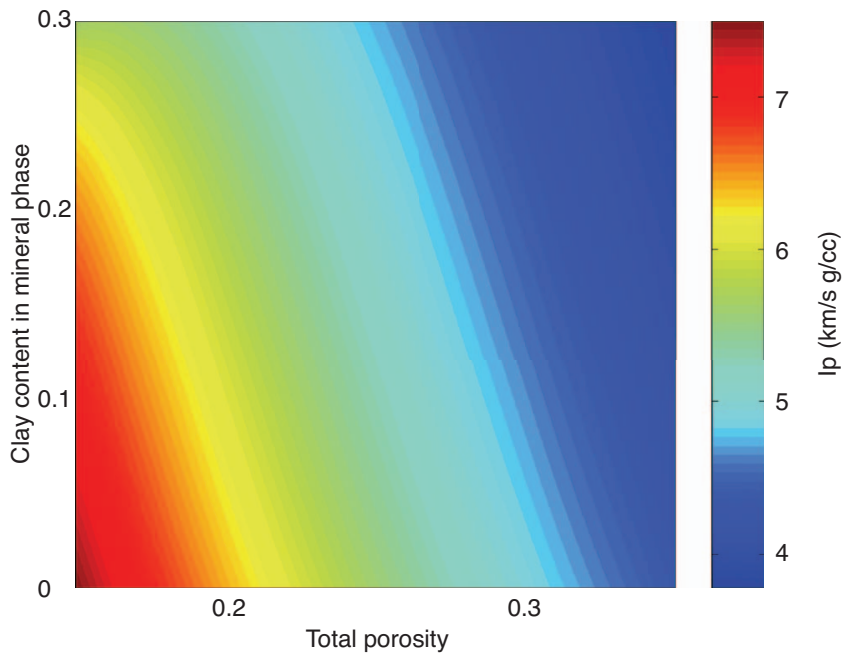

c)

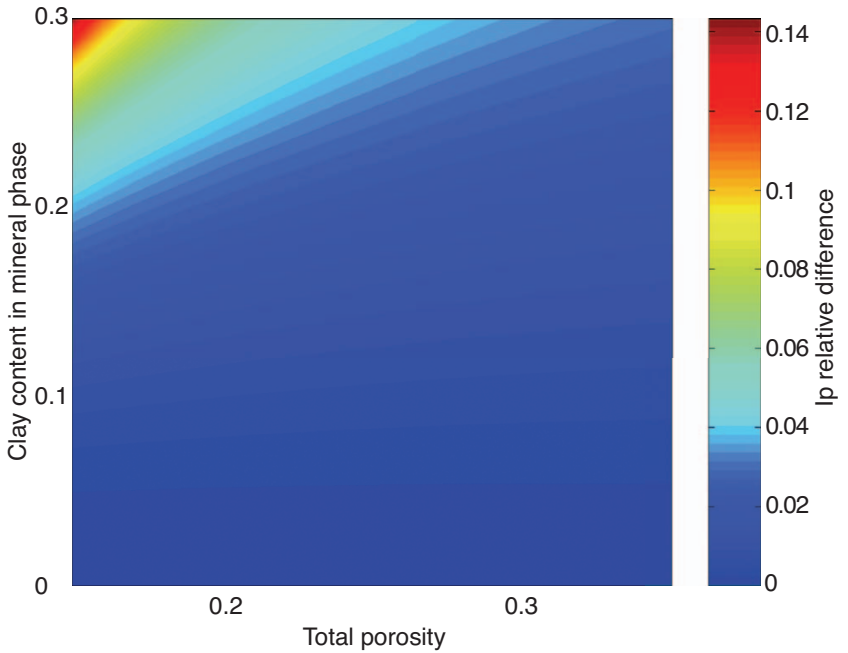

Figure 6. P-wave impedance at partial saturation for a range of porosities and clay contents using (a) the traditional fluid substitution method, (b) the alternative method, and (c) their relative difference for the example discussed in the text. 
a)

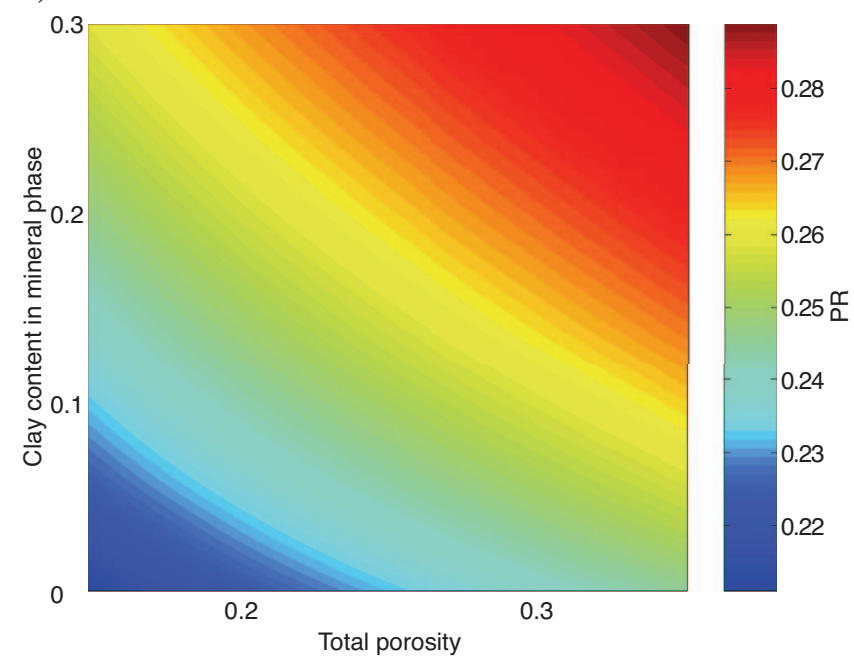

b)

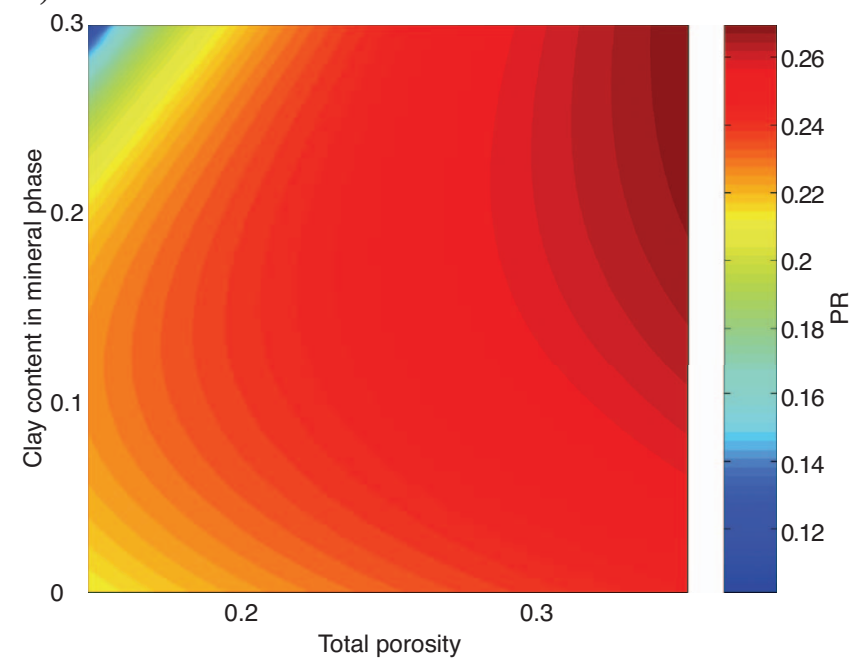

c)

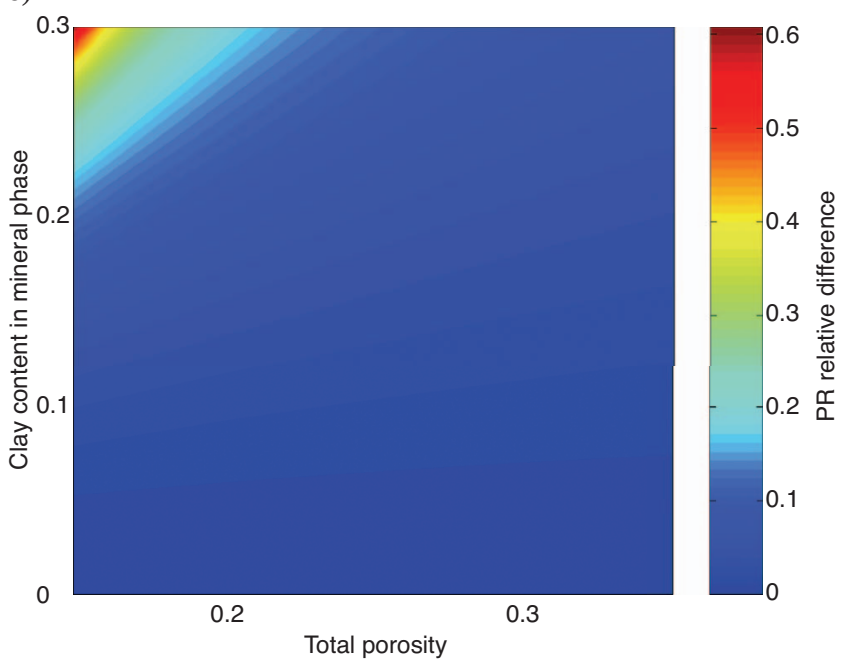

Figure 7. Poisson's ratio at partial saturation for a range of porosities and clay contents using (a) the traditional fluid substitution method, (b) the alternative method, and (c) their relative difference for the example discussed in the text.
Assume that the bulk modulus of the pore fluid (brine) is $2.66 \mathrm{GPa}$ and its density is $1 \mathrm{~g} / \mathrm{cm}^{3}$ (salinity $36,000 \mathrm{ppm}$ at $20 \mathrm{MPa}$ and $70^{\circ} \mathrm{C}$ ). The corresponding compressional and shear moduli of the rock and its Poisson's ratio are shown in Figure 4 for the ranges of total porosity between 0.15 and 0.35 and for clay content in the solid phase between zero and 0.3 .

Assume next that the clay present in the system has an intrinsic porosity of 0.25 and that the corresponding compressional and shear moduli are 10.4 and $1.5 \mathrm{GPa}$, respectively (according to the uncemented sand/shale model). The plots of the total porosity, effective porosity calculated from equation 9 , and difference between the total and effective porosities are shown in Figure 5.

Our goal is to calculate the elastic constants of the rock at partial water saturation of 0.7 , where the hydrocarbon present is predominantly oil (API gravity 35, GOR $2001 / 1$, gas gravity 0.65 ) with a bulk modulus of $0.5 \mathrm{GPa}$ and a density of $0.67 \mathrm{~g} / \mathrm{cm}^{3}$.

For the parameters chosen in this example, the minimum effective porosity of 0.065 is at the minimum total porosity of 0.15 . The minimum effective water saturation is about 0.3 . This saturation becomes zero at the total porosity of about 0.125 , and the effective porosity becomes 0.0375 . At a lower total porosity, the effective water saturation will be negative. This is why we chose 0.15 as a practically reasonable lower bound for the total porosity.

The results for the P-wave impedance and Poisson's ratio using the two fluid substitution methods are shown in Figures 6 and 7, respectively. The largest difference between the results occurs at high clay content and low porosity. There is practically no difference between the two results in high-porosity, clean clastic sediment. This somewhat counterintuitive result (the largest fluid substitution effect occurs at low porosity) is because at the assumed constant total water saturation of 0.7 , the effective water saturation is variable. It is smallest at the lowest porosity and highest clay content (Figure 8) where, as a result, the largest fraction of the water in the effective pore space is replaced by oil.

Let us next use our example to explore the effect of fluid substitution on the seismic amplitude at a pseudowell where a shaley sand layer is placed within a shale interval (Figure 9). We assume that the total porosity of the background wet shale is 0.2 and that the clay content is 0.8 . The total porosity of the shaley reservoir sand is 0.15 ;

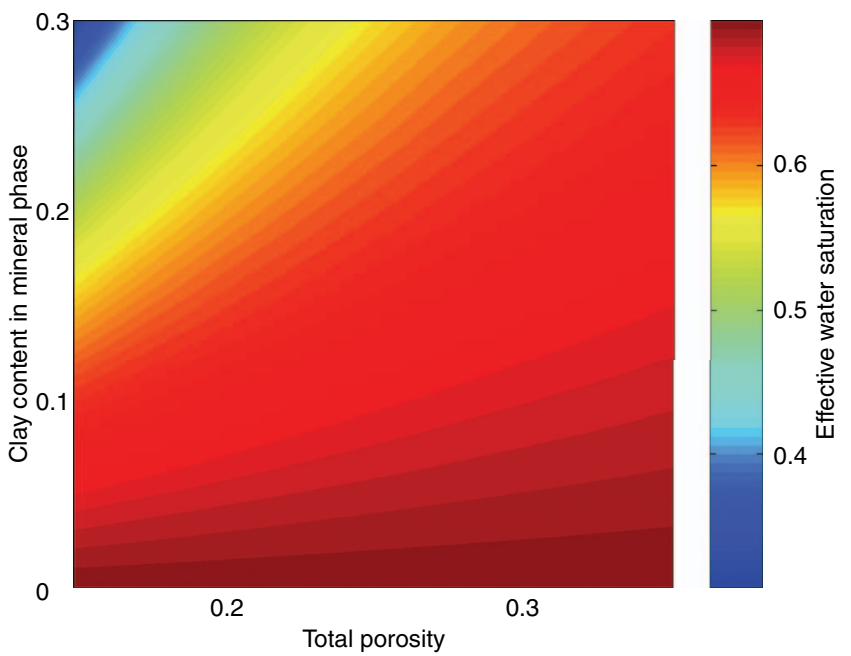

Figure 8. Effective water saturation at a constant total water saturation of 0.8 for a range of porosities and clay contents for the example discussed in the text. 

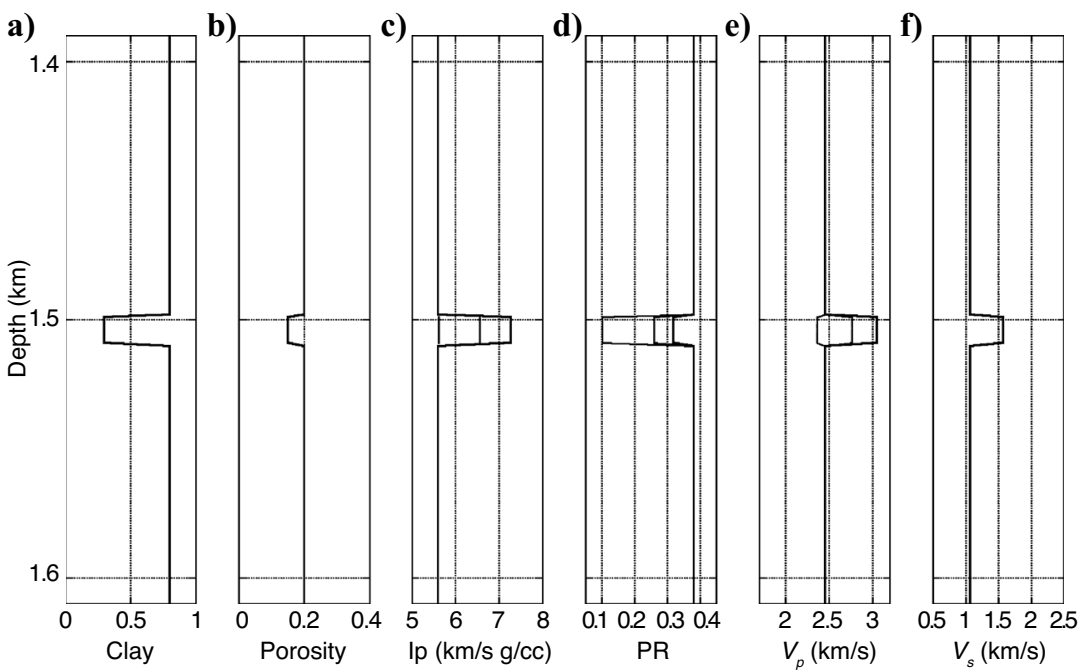

Figure 9. Pseudowell with a single sand layer in a shale background. (a) Clay content, (b) total porosity, (c) P-wave impedance, (d) Poisson's ratio, (e) P-wave velocity, and (f) $\mathrm{S}$-wave velocity. The bold curves in the elastic property frames represent wet sand. The two fine curves represent an oil-saturated sand using the different fluid substitution methods. The higher impedance, Poisson's ratio, and P-wave velocity in the oil sand are derived from the traditional fluid substitution method, while their lower values are from the alternative method. a)

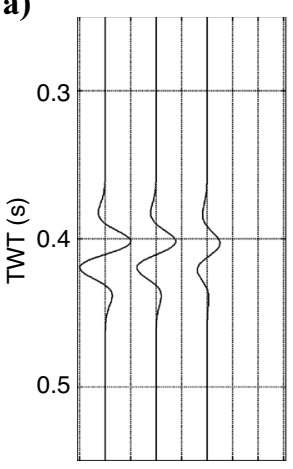

Gather b)

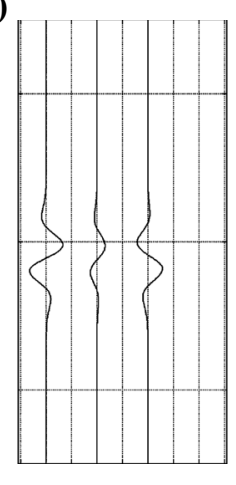

Gather c)

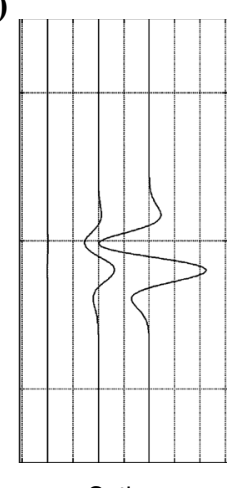

Gather
Figure 10. Synthetic gathers (near-, mid-, and far offsets) at the pseudowell as calculated using a $20-\mathrm{Hz}$ Ricker wavelet. (a) Wet sand, (b) sand with oil using the traditional fluid substitution method, and (c) sand with oil using the alternative fluid substitution method.

that of the clay content is 0.3 . The elastic properties of the interval are calculated according to the uncemented (soft) sand/shale model of Dvorkin and Nur (1996). We also assume that, originally, the sand interval is wet. The purpose of fluid substitution is to predict the seismic response of the same interval with an oil saturation of 0.3 .

The calculated elastic properties of sand with oil are plotted together with the wet-sand properties in Figure 9. We observe dramatic changes in the P-wave impedance and Poisson's ratio depending on the fluid substitution method selected. Although the traditional method predicts a relatively small reduction in the impedance and Poisson's ratio from the wet-sand case, these changes, as calculated using the alternative method, bring the impedance in the oil sand practically to the level of the background (shale) impedance and also drastically reduce the Poisson's ratio of the sand.

The synthetic seismic traces shown in Figure 10 reflect these variations in the elastic properties of the interval. Although we observe a class I AVO response (Rutherford and Williams, 1989) in the wet-sand case, the response becomes class I with phase change for an oil sand whose elastic properties are calculated using the traditional fluid substitution method and, finally, class II where the alternative substitution method is used.

It is important to note that the dramatic differences in the synthetic seismic amplitude responses for the different methods used are because we chose the extreme combination of sand properties (small total porosity and large clay content) that affect fluid substitution results. Much smaller effects are expected in high-porosity clean sand.

\section{CONCLUSION}

Fluid substitution using effective porosity instead of total porosity in sediment may produce noticeable differences between the resulting elastic constants in low-porosity shaley sand. Therefore, where the traditional method cannot explain field observations, the alternative fluid substitution method presented here may be used to ensure a better tie between the well and 3D seismic data or, especially, 4D seismic data. Parameters required by the alternative method include the properties of the shale in the pore space. Although rock physics models are available to calculate the elastic properties of the shale, the choice of both the model used and the parameters of porosity and mineralogy of the shale may be somewhat arbitrary. We recommend selecting and testing geologically consistent ranges of these inputs to estimate the plausible ranges of the elastic response to fluid substitution.

\section{ACKNOWLEDGMENTS}

This work was supported by the U. S. Department of Energy, Stanford Rock Physics Laboratory, and Curtin University of Technology. We thank Per Avseth for his constructive review and Jason Tinder of Rock Solid Images for crucial assistance.

\section{APPENDIX A}

\section{P-WAVE-ONLY FLUID SUBSTITUTION}

Both the traditional method of fluid substitution based on equations 1 and 2 and the alternative method based on equations 14 and 15 require knowledge of the bulk modulus of the fully water-saturated rock $K_{\text {wet }}$, which must be calculated from $V_{p}, V_{s}$, and bulk density $\rho_{b}$ of wet rock as $K_{\text {wet }}=\rho_{b} V_{p}^{2}-(4 / 3) \rho_{b} V_{s}^{2}$.

Often, $V_{s}$ is unavailable or not reliable. To circumvent this difficulty, Mavko et al. (1995) propose an approximate $V_{p}$-only fluid substitution method that uses the compressional modulus $M_{\text {wet }}=K_{\text {wet }}$ - (4/3) $\mu=\rho_{b} V_{p}^{2}$ instead of the bulk modulus $K_{\text {wet }}$. The $V_{p}$-only analogs of equations 1 and 2 are

$$
M_{\mathrm{sat}}=M_{S} \frac{\phi_{t} M_{\mathrm{dry}}-\left(1-\phi_{t}\right) K_{F} M_{\mathrm{dry}} / M_{S}+K_{F}}{\left(1-\phi_{t}\right) K_{F}+\phi_{t} M_{S}-K_{F} M_{\mathrm{dry}} / M_{S}}
$$


and

$$
\begin{aligned}
M_{\mathrm{dry}}= & M_{S} \\
& \times \frac{1-\left(1-\phi_{t}\right) M_{\mathrm{wet}} / M_{S}-\phi_{t} M_{\mathrm{wet}} / K_{W}}{1+\phi_{t}-\phi_{t} M_{S} / K_{W}-M_{\mathrm{wet}} / M_{S}},
\end{aligned}
$$

respectively, where $M$ refers to the compressional modulus and the subscripts have the same meaning as in equations 1 and 2 . The compressional modulus of the solid phase $M_{S}=K_{S}+(4 / 3) \mu_{S}$ can be estimated as Hill's average of the constituents using equations 5-7.

In the alternative method of fluid substitution, the analogs of equations 13 and 14 are

$$
\begin{aligned}
M_{\text {drye }}= & M_{S e} \\
& \times \frac{1-\left(1-\phi_{e}\right) M_{\mathrm{wet}} / M_{S e}-\phi_{e} M_{\mathrm{wet}} / K_{W}}{1+\phi_{e}-\phi_{e} M_{S e} / K_{W}-M_{\mathrm{wet}} / M_{S e}}
\end{aligned}
$$

and

$$
\begin{aligned}
M_{\mathrm{sat}}= & M_{S e} \\
& \times \frac{\phi_{e} M_{\mathrm{drye}}-\left(1-\phi_{e}\right) K_{F e} M_{\mathrm{drye} e} / M_{S e}+K_{F e}}{\left(1-\phi_{e}\right) K_{F e}+\phi_{e} M_{S e}-K_{F e} M_{\mathrm{drye}} / M_{S e}},
\end{aligned}
$$

respectively, where, once again, $M$ refers to the compressional modulus and the subscripts have the same meaning as in equations 13 and 14. The bulk modulus of the fluid $K_{F e}$ is given by equation 15 .

\section{REFRENCES}

Arns, C. H., M. A. Knackstedt, W. V. Pincewski, and E. J. Garboczi, 2002 Computation of linear elastic properties from microtomographic images: Methodology and agreement between theory and experiment: Geophys- ics, 67, 1396-1405.

Berryman, J. G., and G. W. Milton, 1991, Exact results for generalized Gassmann's equations in composite porous media with two constituents: Geophysics, 56, 1950-1960.

Brown, R. J. S., and J. Korringa, 1975, On the dependence of the elastic properties of a porous rock on the compressibility of a pore fluid: Geophysics, 40, 608-616.

Ciz, R., B. Gurevich, A. Siggins, and J. Dvorkin, 2006, Effect of microheterogeneity on the effective stress coefficient for elastic properties of rocks 67th Annual Conference and Exhibition, EAGE, Extended Abstracts, Paper P045.

Dvorkin, J., and A. Nur, 1996, Elasticity of high-porosity sandstones: Theory for two North Sea data sets: Geophysics, 61, 1363-1370.

Gassmann, F., 1951, Über die Elastizität poroser Medien: Vierteljahrsschrift der Naturforschenden Gesellschaft Zürich, 96, 1-23.

Goldberg, I., and B. Gurevich, 1998, A semi-empirical velocity-porositydepth model for petrophysical interpretation of $\mathrm{P}$ and $\mathrm{S}$ velocities: Geophysical Prospecting, 46, 271-285.

Gurevich, B., and J. Carcione, 2000, On Gassmann modeling of acoustic properties of sand-clay mixtures: Pure and Applied Geophysics, 157, 811 827.

Hashin, Z., and S. Shtrikman, 1963, A variational approach to the elastic behavior of multiphase materials: Journal of Mechanics and Physics of Solids, 11, 127-140.

Hill, R., 1952, The elastic behavior of crystalline aggregate: Proceedings of the Physical Society of London, A65, 349-354.

Holt, R. M., and E. Fjaer, 2003, Wave velocities in shale, a rock physics model: 64th Annual Conference and Exhibition, EAGE, Extended Abstracts, Paper C13.

Holt, R. M., E. Fjaer, and E. Rzayev, 2004, P- and S-wave velocities in shales: Experiments and a model: North American Rock Mechanics Symposium, American Rock Mechanics Association, Paper 04-543.

Mavko, G., C. Chan, and T. Mukerji, 1995, Fluid substitution: Estimating changes in $V_{p}$ without knowing $V_{s}$ : Geophysics, 60, 1750-1755.

Mavko, G., T. Mukerji, and J. Dvorkin, 1998, Rock physics handbook: Cambridge University Press.

Raymer, D. S., E. E. Hunt, and J. S. Gardner, 1980, An improved sonic transit time-to-porosity transform: 21 st Annual Meeting, Society of Petrophysicists and Well Log Analysts, Paper P.

Rutherford, S. R., and R. H. Williams, 1989, Amplitude-versus-offset variations in gas sands: Geophysics, 54, 680-688.

Smith, T. M., C. H. Sondergeld, and C. S. Raiz, 2003, Gassmann fluid substitutions: A tutorial: Geophysics, 68, 430-440.

White, J. E., 1983, Underground sound: Application of seismic waves: Elsevier Science Publ. Co., Inc.

Wood, A. B., 1941, A textbook of sound: G. Bell and Sons Ltd. 